\title{
Evaluation of armor stone performance and deterioration
}

\author{
D. W. Harrelson ${ }^{1}$, M. Zakikhani ${ }^{1}$, J. G. Tom ${ }^{2}$, J. A. Kissane ${ }^{2}$, \\ M. K. Allis ${ }^{3}$ \& J. E. Kolber ${ }^{4}$ \\ ${ }^{1}$ The U.S. Army Engineer Research and Development Center, USA \\ ${ }^{2}$ U.S. Army Corps of Engineers, Chicago District, USA \\ ${ }^{3}$ U.S. Army Corps of Engineers, Detroit District, USA \\ ${ }^{4}$ U.S. Army Corps of Engineers, Buffalo District, USA
}

\begin{abstract}
Evaluating long-term performance and deterioration of armor stones are essential for maritime structures to protect harbors or navigable areas. Armor rocks are impacted by the natural elements such as seasonal weather, and repeated cycles of temperature (e.g., flowing water, wetting and drying, wave action, freeze and thaw, etc.). The rock's behavior in the field may vary greatly from the controlled laboratory test results. The design process for the determination of optimal armor stone sizes is complex. Numerous investigators have studied the development of relationships for the minimum stable weight of a rubble-mound armor unit for given wave conditions. The main objective of this study has been to evaluate major factors involved in armor stone durability. To consider the combined effects of environmental stresses on armor stone, several testing procedures have been developed to evaluate the performance of stone subjected to both freezing and thawing and wetting and drying. Long-term performance or deterioration of armor stones have been quantitatively monitored and characterized by the changes in dimensions measured. A degradation numerical model has been developed that relates the laboratory test results to the armor stone mass reduction at the project site. The paper describes the latest results and developed tools for the armor stone evaluations. New approaches are introduced that may be used to evaluate the quality and durability with reference to breakage and integrity.
\end{abstract}

Keywords: hydrodynamic forces, response of structures, fluid dynamics, armor stone durability, great lakes, numerical modeling, laboratory testing. 


\section{Introduction}

Degradation of armor stone placed in coastal navigation areas in the United States Great Lakes has occurred as result of a number of interacting and interrelated natural factors such as seasonal weather changes, and repeated cycles of temperature (e.g., flowing water, wetting and drying, wave action, freeze and thaw, etc.). This study was proposed to evaluate the importance of various factors in armor stone durability, consider the relevance of test procedures and possibly develop scaling factors and test methods that will be more representative of the materials used. It also provides guidelines for the personnel involved in source selection activities, so they may be more consistent across the organization. The research provides a better understanding of the relationship between the standard tests used in stone specifications and the performance of stone in the structures. Several investigations have developed standard field and laboratory procedures for careful comparisons of stone of similar, if not identical, properties. Previous investigations have been directed at considering the causes, based on the comparison of different types of stone used in different structures. Much of the testing performed on stone submitted as material for erosion protection is based on the protocols used to evaluate concrete aggregate and sub-grade stone materials - that is, tests designed for use on material orders of magnitude smaller than armor stone of several tons. Acceptance of sources is then based on a combination of test results, performance records, and quarry inspections.

The main objectives of this study are to evaluate factors involved in armor stone durability and investigate the effects of scaling on test results using samples of various rock types used in the United States Great Lakes coastal projects. As a secondary objective, beyond evaluating testing, the results of this study and the previous work on armor stone durability would be used to develop guidelines for future armor stone selection procedures with respect to ranking of stone types, excavation methods and geologic characteristics of the materials available within a region. These guidelines could also be considered when developing documents such as "Engineering Considerations during Construction", for use in evaluating proposed sources.

After review of existing literature and data and consultation with each of the USACE Districts in the Great Lakes region (Buffalo, Chicago and Detroit Districts), three projects, Burns Harbor, Cleveland Harbor and Keweenaw Waterway have been selected for inclusion in this study. Representative sections of each project have been selected for evaluation of armor stone performance. Index stones have been placed in the structures and monitored over the study period. Samples from quarries used in shoreline projects have been designated and tested using existing ASTM or testing protocols contained in existing stone specifications that stipulate the size of samples. These test results have been compared to tests of larger size samples to consider the effects of scale. In addition, larger samples have been placed in structures so that they may be subjected to the conditions that cause weathering of armor stone and the results of their performance have been compared to the laboratory results. To consider 
the combined effects of environmental stresses on armor stone, testing have been done to evaluate the performance of stone subjected to both freezing and thawing and wetting and drying. This testing has been performed on the same samples rather than independently, as is more typically done. The combined testing realistically simulates the environmental conditions in the structure. In addition, by including stone of different rock types (i.e. igneous, metamorphic and sedimentary), the relative scaling effects can be evaluated to determine whether the scaling factors, if they exist, are constant or variable by rock type. After evaluating monitoring data, a determination can be made as to the relative comparability of scaled laboratory test results and material durability in coastal projects in the Great Lakes, and recommendations made as to the appropriate laboratory tests for future stone specifications.

\section{Field evaluation and design}

\subsection{Design}

The design process for the determination of riprap and armor stone sizes is complex, particularly for the various geometries of channel protection, and is beyond the scope of this article. Various factors must be considered in order to fully understand how the design parameters have an indirect effect on stone performance, and two of the more useful of the design equations will be reviewed to demonstrate the influence of these factors.

Hudson [4] developed the best known of the design equations for determination of acceptable armor stone size to resist damage from a given wave system based on hydraulic modeling studies. The equation is as follows:

$$
W=H^{3} w_{r} / K_{d}(S r-1) \cot \theta
$$

where $W$ is the weight of the armor unit, $H$ is the average wave height of the highest 10 percent of all waves, $w_{r}$ is the unit mass of the stone, $K_{d}$ is a damage coefficient, $S_{r}$ is the specific gravity of the stone, and $\theta$ is the angle of the slope of the armor stone.

Hudson [4] presents the results of an extensive series of experiments conducted to obtain basic information on the stability, $K_{d}$ of rubble-mound breakwaters. These equations along with other design formulations have been used in this study.

\section{Laboratories and experimental test}

\subsection{Samples}

To determine qualified samples for this study, several quarry sites that have historically supplied material for shoreline protection in and around the Great Lakes Region were visited. The five samples received represent two of five material types proposed for the entire investigation. Samples identified as 
MCNP 1, 2, 3 are stone discard pieces from stones placed in the field for monitoring from the Hayton Quarry. Each sample consisted of two slabs, each approximately $4-\mathrm{ft}$ by $6-\mathrm{ft}$ by $1 \mathrm{ft}$, from the original stone and was assigned Concrete and Materials Branch Serial No. 070103, 070104, and 070105, respectively. The smaller, thinner slabs are further identified as Slab A and the larger thicker, more massive slabs are further identified as Slab B (Figure 1). Samples identified as MCNP 4 and 5 are also discard pieces from stones placed in the field from Valders Quarry. These samples also consisted of two slabs, each approximately $4-\mathrm{ft}$ by $6-\mathrm{ft}$ by $1-\mathrm{ft}$, and was assigned CMB Serial No. 070106 and 070107 , respectively. The samples proposed for Phase I of this investigation are the CMB Serial No. 070104 (MCNP-2) and 070105 (MCNP-3).
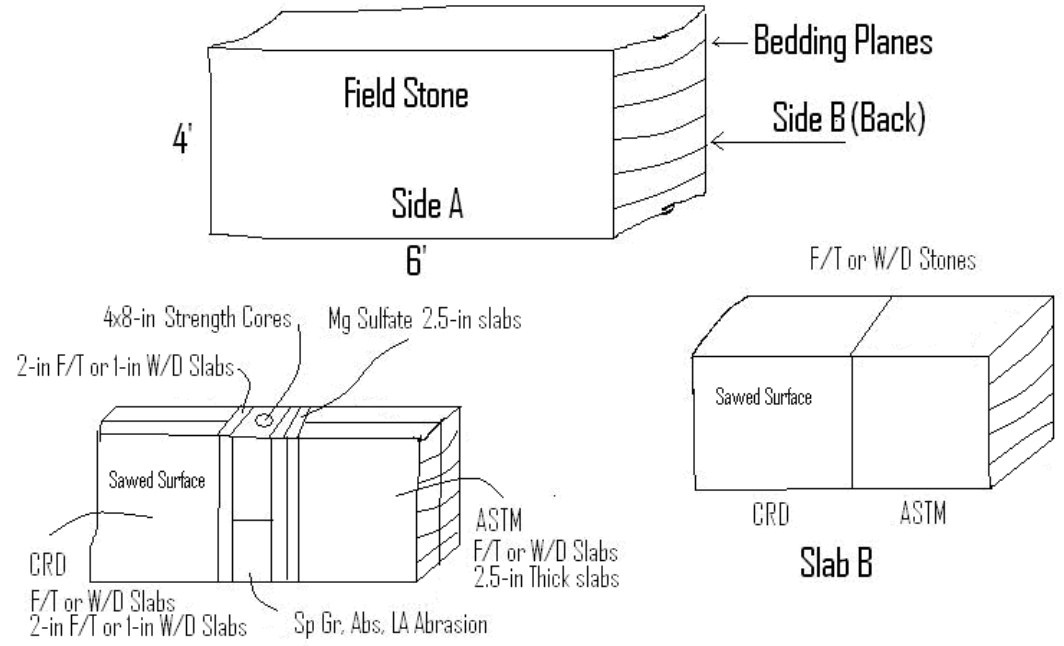

Slab B

Slab A

Figure 1: Drawing of the cutting lines and test specimens.

\section{Degradation model for armor stone}

\subsection{Estimation of degradation rate}

Latham [6] provided a simple approach to estimate reduction in armor rock weights as results of several environmental and other impacts. Factors affecting the degradation rate are the intrinsic material properties of the rock source, the production-influenced geometric properties of the armors tine, the environmental boundary conditions at the coastal site, and the armor layer design concepts used. The parameters affecting stone degradation rates are provided in Table 1.

To determine degradation rate for a given armor stone, a sample of the material is tested in an abrasion mill simulation of the wear process. This provides a graph of weight versus laboratory time. Laboratory time is converted 


\subsection{Application of degradation model}

The rock samples are used in the abrasion mill to create plot of fractional weight loss versus revolutions (Figure 2). This graph gives the typical loss in asperity roughness observed in milling tests. The equivalent wear time factor, $X$ is calculated as product of all ratings.

$$
X=\sum_{\mathrm{i}}^{9} X_{i}
$$

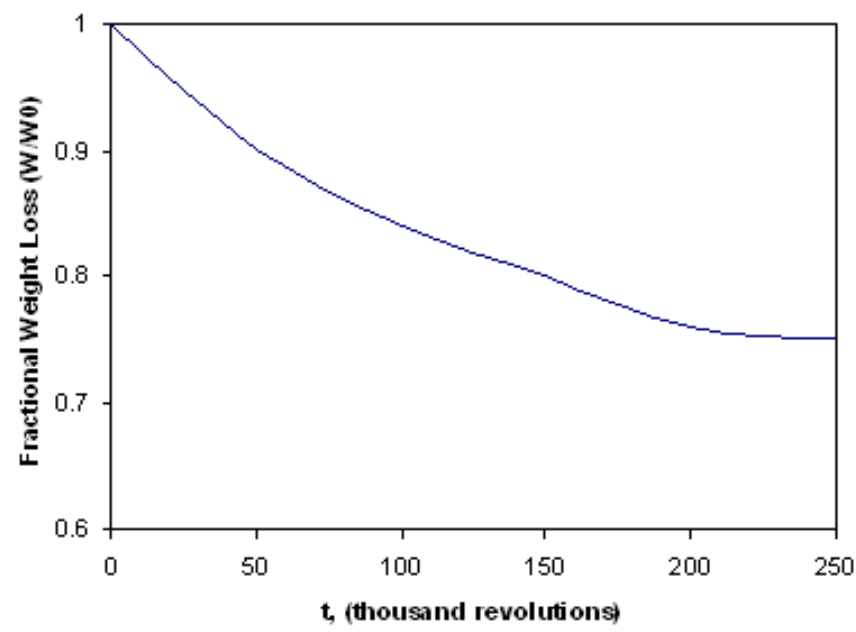

Figure 2: Abrasion mill test results for specific type of armor stone.

Table 2: Example site situation summary.

\begin{tabular}{|l|l|l|l|}
\hline \multicolumn{2}{|l|}{} & \multicolumn{2}{l|}{ Ratings } \\
\hline \multicolumn{2}{|l|}{ Parameter } & (i) & (ii) \\
\hline$X_{1}$ & size & 0.72 & 0.84 \\
\hline$X_{2}$ & grading & 1.0 & 1.2 \\
\hline$X_{3}$ & shape & 1.5 & 1.5 \\
\hline$X_{4}$ & wave energy & 2.0 & 2.0 \\
\hline$X_{5}$ & zone & 1.0 & 1.0 \\
\hline$X_{6}$ & climate & 0.2 & 1.0 \\
\hline$X_{7}$ & attrition & 1.0 & 1.0 \\
\hline$X_{8}$ & $\begin{array}{l}\text { concentration } \\
\text { of attack }\end{array}$ & 1.5 & 1.0 \\
\hline$X_{9}$ & $\begin{array}{l}\text { block } \\
\text { mobility }\end{array}$ & 1.0 & 2.0 \\
\hline$X$ & & 0.6 & 6.0 \\
\hline
\end{tabular}


The value $X$ is then used to convert number of years in service to thousands of revolutions in mill. Using Figure 1 and the number of revolutions, $W / W_{0}$ will be estimated and finally the reduction in weight will be calculated.

As an example input parameters for two site situations are given in Table 2 (Latham [6]): (i) for a 3 ton, basalt in tropical climate, medium grading, dynamic design, and (ii) for 4.5 ton, temperate climate, narrow grading, static design.

\subsection{Characterization of rock heterogeneity}

Liu et al. [8] describes a statistical approach (homogeneity index) to characterize the heterogeneity in rock. According to this article, the Weibull distribution (Weibull [9]; Hudson and Fairhurst, [5]) describes very well the experimental data for the distribution of microstructures within rock. Therefore, the Weibull statistical distribution is used to characterize the rock heterogeneity. The Weibull distribution may be simplified as:

$$
\mathrm{Q}(\sigma)=\int_{0}^{\sigma} \mathrm{P}(\mathrm{x}) \mathrm{dx}=1-\exp \left[-\left(\frac{\sigma}{\sigma_{0}}\right)^{\mathrm{m}}\right]
$$

where $\mathrm{Q}$ is a simplified form of Weibull distribution, $\sigma$ is the elemental parameter (MPa), $\mathrm{P}$ is the Weibull probability density function, $\mathrm{m}$ is the shape parameter describing the scatter of $\sigma$ and describes the heterogeneity of rock, and $\sigma_{0}$ is the mean value of the physical-mechanical parameters of the specimen (elemental parameter).

The most recommended method for calculating the homogeneous index $\mathrm{m}$ (Curtis and Juszczyk, [2]; Davies, [3]) is to rank strength $(\sigma)$ data from smallest to largest and the assignment of respective $Q(\sigma)$ values according to the following:

$$
\mathrm{Q}(\sigma)=\frac{\mathrm{i}}{\mathrm{N}+1}
$$

Where $\mathrm{i}$ is the rank and $\mathrm{N}$ is the total number of specimens. According to Equation 3, the Weibull distribution can be liberalized into the following form:

$$
\mathrm{y}=\ln \left[\ln \left(\frac{1}{1-\mathrm{Q}(\sigma)}\right)\right]=\mathrm{m} \ln \sigma-\mathrm{m} \ln \sigma_{0}=\mathrm{Ax}+\mathrm{B}
$$

where $\mathrm{y}=\ln \ln \{1 /[1-\mathrm{Q}(\sigma)]\}, \mathrm{A}=\mathrm{m}, \mathrm{x}=\ln \sigma$, and $\mathrm{B}=\mathrm{m} \ln \sigma_{0}$. With reference to this equation, a plot of $\ln \sigma$ against $\ln \ln \{1 /[1-\mathrm{Q}(\sigma)]\}$ gives the line-relationship and the slope of the line is the homogeneous index $\mathrm{m}$. The best estimate of the homogeneous $m$ may be obtained using the linear least squares (LLS) techniques (Davies [3]): 


$$
\mathrm{m}=\mathrm{A}=\frac{\mathrm{n} \sum \mathrm{xy}-\sum \mathrm{x} \sum \mathrm{y}}{\mathrm{n} \sum \mathrm{x}^{2}-\left(\sum \mathrm{x}\right)^{2}}
$$

where $\sum, \mathrm{x}$ and $\mathrm{y}$ in the equations are abbreviations for $\sum_{\mathrm{i}=1}^{\mathrm{n}}, \mathrm{x}_{\mathrm{i}}$ and $\mathrm{y}_{\mathrm{i}}$, respectively.

One of the attractive aspects of the Weibull distribution is the presence of the shape parameter, which allows this function to take a wide variety of shapes. For $\mathrm{m}=1$; this distribution is exponential; at about $\mathrm{m}=1.5$; the distribution is nearly log-normal; and at about $\mathrm{m}=4$, it closely approximates a normal distribution. Since the shape parameter $\mathrm{m}$ is a measure of the element parameter variability, it can be considered as a homogeneity index. The larger the index $\mathrm{m}$ is, the more homogeneous is the rock. When $m$ tends to infinity, the variance tends to zero and an ideal homogeneous rock is obtained.

\subsection{Estimation of freeze-thaw intensity}

The intensity of freezing and thawing depends on the freezing temperature, the duration of the freezing cycle, the available moisture, the slope direction (geographic area properties), degree of saturation, and permeability (rock properties). Lienhart [7] describes the following technique (formulation) to measure the intensity of freeing and thawing.

$$
\mathrm{MNFC}=\sum_{\mathrm{i}=1}^{12}(\operatorname{MNMaxT}-\mathrm{MNMinT})_{\mathrm{i}}
$$

where:

$\mathrm{MNFC}=$ Mean number of freezing cycle (days/Year)

MNMaxT $=$ Mean number of days of maximum temperature of 32F and below for each month

MNMinT=Mean number of days of minimum temperature of 32F and below for each month

Since the amount of moisture affects the freeze-thaw durability, the mean number of days of precipitation of 0.01 in or more for those months in which freezing cycle days occur (MNDP) was also calculated. The percent days of precipitation of 0.01 inch or more during the freezing cycle month (PP) is given as:

$$
\mathrm{PP}=\frac{\text { MNDP }}{(\text { MNMaxT }- \text { MNMinT })}
$$

The moist freeze-thaw index (MFTI) then may be calculated from the following equation as:

$$
\text { MFTI }=\sum_{\mathrm{i}=1}^{12}((\text { MNMaxT }- \text { MNMinT }) \times P P)_{i}
$$

Lienhart [7] used data from 254 weather stations from the National Oceanic and Atmospheric Administration (NOAA) and plotted the calculated moist freeze-thaw index for the contiguous United States (Figure 3). 


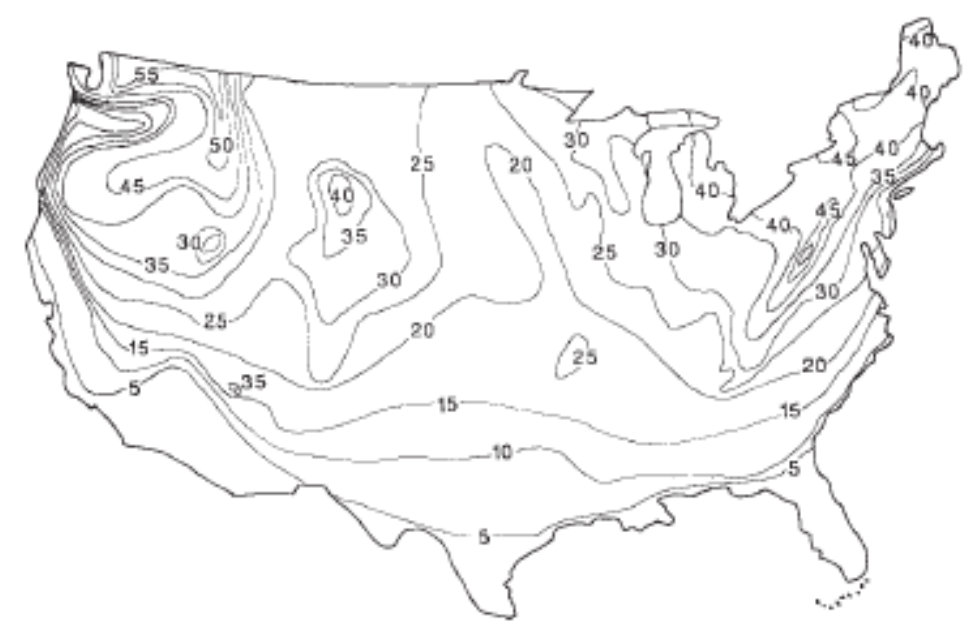

Figure 3: Isoline map of the moist freeze-thaw index for the United States.

\section{Results}

The project described here is a multi-year research effort. To date we have completed part of the study. The results presented here are not conclusive. The reader should look for future publications of this research project. Currently, ten index stones have been placed at Keweenaw Waterway and eight index stones at Cleveland Harbor. Placement of index stones at Burns Harbor is scheduled for summer of 2008. The Keweenaw samples consist of ten stones, five lime stones, two quartzites, two granites and one cast concrete block. All Keweenaw index stones (except the cast concrete) were cut into roughly rectangular shapes producing a 5-6 ton stone. The concrete block was cast into approximately the same dimensions as the other cut stones. Cleveland Harbor's index stones consist four cut stones; two sandstones and two lime stones and four cast concrete blocks (with varying concrete mixtures). These stones also rectangular in shape average about 9 tones in weight. They were placed in Cleveland Harbor in September of 2007, and monitoring is set to begin in spring 2008.

The index stones placed in Keweenaw Waterway and Cleveland Harbor are currently being monitored for rock mass-loss and degradation. Additionally, specific macroscopic features being monitored include vugs, stylolites and fossils in the limestones and fractures, joints and cracks in the quartzite and granites. Petrographic analysis of various microscopic features is continuing as part of the laboratory efforts.

A total of four rounds of monitoring have been conducted at Keweenaw Waterway. Field observations on these samples indicate that in general the stones are weathering from the edges inward towards the center of the stone. The cast concrete block has shown the most weathering with measurable deterioration and mass-loss beginning along its edges (and some corners) that is progressing towards the center of the stone. This trend is less evident in the other 
index stones, but four rounds of observations indicate that although stone deterioration and mass-loss is less than for the concrete block, it is still progressing in a similar manner.

The limestones, quartzites, and granites have had little measurable rock massloss and display no clear trends, but numerous macroscopic features including fractures and joints on all rock types and vugs and stylolites found in the limestones are being monitored for change and a time line for a stone deterioration index is underway.

Several of the Keweenaw index stones displayed ice abrasion during their first winter (2006). This abrasion occurred mainly as scoring of the rock at its waterline due to ice movement. One stone, a quartzite, was moved (displaced to the toe of the structure) during a large storm event in October of 2006, but recovered and replaced on the structure in August 2007.

The field observations and laboratory testing will be used to construct a "stone durability index model" that will be useful in determining the life cycle of large (type A) armor stone. This model will integrate field observations and laboratory testing into a common index that will predict percent rock mass-loss and deterioration rate.

\section{References}

[1] Curtis, D. C., Stability of minerals in surface weathering reactions: A general thermochemical approach: Earth Surface Processes, v. I: p.63-70, 1976.

[2] Curtis, R.V., Juszczyk, A.S., Analysis of strength data using two- and threeparameter Weibull models. J. Mater. Sci. 33, 1151-1157, 1988.

[3] Davies, I.J., 2001. Empirical correction factor for the best estimate of Weibull modulus obtained using linear least square analysis. J. Mater. Sci. Lett. 20, 997- 999, 2001.

[4] Hudson, R. Y., Design of Quarry-Stone Cover Layers for Rubble-Mound Breakwaters: U.S. Army Engineer Waterways Experiment Station Research Report No. 2-2, Vicksburg, MS, 39 p., 1958.

[5] Hudson, J.A., Fairhurst, C., Tensile strength, Weibull's theory and a general statistical approach to rock failure. The Proceedings of the Civil Engineering Materials Conference, Held in Southampton 1969, pp. 901-904, 1069.

[6] Latham, J-P., "Degradation model for rock armour in coastal engineering", Quarterly Journal of Engineering Geology, 24, 101-118, 1991.

[7] Lienhart, D.A., The geographic distribution of intensity and frequency of freeze-thaw cycles. Bulletin of the Association of Engineering Geologists. Vol. 25, No. 4, p. 465-469, 1988.

[8] Lui H.Y., M. Roquete, S.Q. Kou, P.-A. Lindqvist, Characterization of rock heterogeneity and numerical verification, Engineering Geology 72 (2004) 89-119, 2004.

[9] Weibull, W., A statistical distribution function of wide applicability. J. Appl. Mech., 293-297, 1951. 